\title{
On the description of exotic hadron states within QCD sum rules
}

\author{
Wolfgang Lucha ${ }^{1, a}$ and Dmitri Melikhov ${ }^{1,2,3, b}$ \\ ${ }^{1}$ Institute for High Energy Physics, Austrian Academy of Sciences, Nikolsdorfergasse 18, A-1050 Vienna, \\ Austria \\ ${ }^{2}$ Faculty of Physics, University of Vienna, Boltzmanngasse 5, A-1090 Vienna, Austria \\ ${ }^{3}$ D. V. Skobeltsyn Institute of Nuclear Physics, M. V. Lomonosov Moscow State University, 119991, Moscow, \\ Russia
}

\begin{abstract}
Revisiting the QCD sum-rule description of exotic hadron states, we argue that, in order to arrive at trustable analyses of, for instance, strong decay widths of multiquarks, it is inevitable to adopt the QCD sum-rule approach beyond the leading perturbative level.
\end{abstract}

\section{Incentive: analytic nonperturbative approach to multiquark bound states}

Exotic hadrons are colour-singlet multiquark bound states not composed just of a quark-antiquark pair or of three (anti-) quarks. Candidates for this kind of multiquark bound states, specifically, for mesonic four-quark states, so-called tetraquarks, and for baryonic five-quark states, so-called pentaquarks, have been observed by experiment. Here, we would like to add a few general considerations on peculiarities and opportunities encountered in an analysis of exotic hadrons by means of QCD sum-rule techniques.

After recalling briefly the conceptual foundations and assumptions of the QCD sum-rule approach, we point out a crucial qualitative difference between the treatment of ordinary hadrons and the study of exotic hadrons and we explore options for disclosing these states' structure from their decay constants.

\section{QCD sum-rule formalism: the example of two-point correlation functions}

QCD sum rules [1-3] are analytic relationships between observable properties of a given hadron $H$ and the basic parameters of the quantum field theory of the strong interactions, quantum chromodynamics. The starting point of their derivation is a conveniently chosen operator which interpolates the hadron $H$ under study, i.e., which has a non-vanishing matrix element when being sandwiched between the QCD vacuum state, $|\Omega\rangle$, and the hadron's state $|H\rangle$. Usually, this interpolating operator is formulated as some current, defined in terms of the fundamental degrees of freedom of QCD, quarks and gluons, and, upon suppressing notationally the behaviour under Lorentz transformations, henceforth generically denoted by $j(x)$. The magnitude of its vacuum-hadron amplitude may be characterized by a decay constant, $f_{H}$ :

$$
\langle\Omega|j(0)| H\rangle \propto f_{H} \neq 0 .
$$

The QCD sum rules then result from the evaluation of appropriate $n$-point correlation functions of such interpolating currents in parallel at the level of hadrons and at the level of the QCD degrees of freedom.

\footnotetext{
ae-mail: Wolfgang.Lucha@oeaw.ac.at

be-mail: dmitri_melikhov@gmx.de
} 
The clearly least intricate case is the two-point correlation function, given by the Fourier transform of the (QCD-) vacuum expectation value of the time-ordered product of two $H$-interpolating currents $j$,

$$
\Pi\left(p^{2}\right) \equiv \mathrm{i} \int \mathrm{d}^{4} x \exp (\mathrm{i} p x)\left\langle\Omega\left|\mathrm{T}\left(j(x) j^{\dagger}(0)\right)\right| \Omega\right\rangle \text {. }
$$

Given the validity of the operator product expansion hypothesized by Wilson [4, 5], a nonlocal product of operators can be converted into a series of (linearly independent) local operators $O_{n}, n=0,1,2, \ldots$, with Wilson coefficient functions $C_{n}$, depending on space-time distances, $x^{2}$, and an energy scale $\mu$ that defines the distinction of perturbative and nonperturbative contributions to vacuum expectation values:

$$
\mathrm{T}\left(j(x) j^{\dagger}(0)\right)=\sum_{n=0}^{\infty} C_{n}\left(x^{2}, \mu\right): O_{n}(x=0, \mu):=C_{0}\left(x^{2}, \mu\right)+\sum_{n=1}^{\infty} C_{n}\left(x^{2}, \mu\right): O_{n}(x=0, \mu):
$$

Herein, colons emphasize the free-field normal ordering of the composite operators $O_{n}(x, \mu)$, and in the second equality the unit operator has been highlighted by separation from the sum over local operators. The correlation function $\Pi\left(p^{2}\right)$ reflects the latter general expression of the operator product expansion: $\Pi\left(p^{2}\right)$ consists of a portion $\Pi_{\text {pert }}\left(p^{2}, \mu\right)$ entirely determined by perturbative quantum chromodynamics,

$$
\Pi\left(p^{2}\right)=\Pi_{\text {pert }}\left(p^{2}, \mu\right)+\sum_{n=2}^{\infty} \frac{C_{n}}{\left(p^{2}\right)^{n}}\left\langle\Omega\left|: O_{n}(0, \mu):\right| \Omega\right\rangle,
$$

and a series of terms controlled by the vacuum expectation values of all gauge-invariant local operators $O_{n}(x, \mu)$ that are constructible in terms of the QCD fields and their derivatives, the vacuum condensates

$$
\langle\Omega|: O(0, \mu):| \Omega\rangle \neq 0,
$$

which assume non-vanishing values as a consequence of the nontrivial nature of the QCD vacuum, $|\Omega\rangle$. Allowing for complex values of the momentum variable $p^{2}$, taking advantage of the analytic behaviour of $\Pi\left(p^{2}\right)$ as function of $p^{2}$, performing in the complex- $p^{2}$ plane both the analytic continuation of $\Pi\left(p^{2}\right)$ and a contour integration, exploiting the Cauchy integral formula, and assuming an appropriately rapid decrease of $\Pi\left(p^{2}\right)$ in the limit $\left|p^{2}\right| \rightarrow \infty$ of the absolute value $\left|p^{2}\right|$ enables us to represent the correlation function $\Pi\left(p^{2}\right)$ in the guise of a dispersion relation involving a spectral density generically called $\rho(s)$ :

$$
\Pi\left(p^{2}\right)=\int \frac{\mathrm{d} s}{s-p^{2}} \rho(s) .
$$

At the hadron level, inserting a complete set of intermediate hadron states into our vacuum expectation value and singling out the hadronic state of interest, i.e., the ground state of mass $M_{H}$, by subsuming all excited and continuum states in a "continuum" contribution, $\rho_{\text {cont }}(s)$, yields the hadron spectral density

$$
\rho_{\text {hadr }}(s)=f_{H}^{2} \delta\left(s-M_{H}^{2}\right)+\rho_{\text {cont }}(s) .
$$

At the QCD level, the spectral density inherits the partition into perturbative and nonperturbative parts:

$$
\rho_{\mathrm{QCD}}(s)=\rho_{\text {pert }}(s, \mu)+\sum_{n=2}^{\infty} C_{n} \delta^{(n)}(s)\left\langle\Omega\left|: O_{n}(0, \mu):\right| \Omega\right\rangle .
$$

At the hadron level, removal of possible subtraction terms and reduction of the impact both of hadronic excitations above the ground state and of the hadronic continuum may be accomplished by applying, to the correlation function, a Borel transformation $\mathcal{B}_{\tau}$ to a new variable, the Borel parameter $\tau$, defined by

$$
\Pi\left(p^{2}\right) \stackrel{\text { Borel }}{\longrightarrow} \Pi(\tau)=\mathcal{B}_{\tau}\left[\Pi\left(p^{2}\right)\right] \equiv \lim _{\substack{2 \\ p^{2} \rightarrow-\infty \\ n \rightarrow \infty \\-p^{2} / n=1 / \tau}} \frac{\left(-p^{2}\right)^{n+1}}{n !}\left(\frac{\mathrm{d}}{\mathrm{d} p^{2}}\right)^{n} \Pi\left(p^{2}\right) .
$$


Of particular interest are the Borel transforms of poles at a point $a$, emerging from the above definition:

$$
\mathcal{B}_{\tau}\left[\frac{1}{\left(a-p^{2}\right)^{k}}\right]=\frac{\tau^{k-1}}{(k-1) !} \exp (-a \tau), \quad k \in \mathbb{N}_{>0} \equiv\{1,2,3, \ldots\}
$$

The "continuum" spectral density $\rho_{\text {cont }}(s)$ vanishes below some physical threshold $s_{\text {phys }}$, defined by the location of the lowest-lying hadron excitation or the onset of the hadronic continuum. The perturbative spectral density $\rho_{\text {pert }}(s, \mu)$ vanishes below some "theoretical" threshold $s_{\text {th }}$. For an interpolating current formed by a quark and an antiquark, of masses $m_{1}$ and $m_{2}$, respectively, $s_{\mathrm{th}}$ is given by $s_{\mathrm{th}}=\left(m_{1}+m_{2}\right)^{2}$. Therefore, referring by $\Pi_{\mathrm{NP}}(\tau, \mu)$ to the Borel transform of the total nonperturbative contribution to the QCD spectral density $\rho_{\mathrm{QCD}}(s)$, our Borel-transformed (or "Borelized") correlation function $\Pi(\tau)$ reads

$$
\begin{aligned}
\Pi(\tau) & =\int \mathrm{d} s \exp (-s \tau) \rho(s) \\
& =f_{H}^{2} \exp \left(-M_{H}^{2} \tau\right)+\int_{s_{\text {phys }}}^{\infty} \mathrm{d} s \exp (-s \tau) \rho_{\text {cont }}(s) \quad \text { (at hadron level) } \\
& =\int_{s_{\text {th }}}^{\infty} \mathrm{d} s \exp (-s \tau) \rho_{\text {pert }}(s, \mu)+\Pi_{\mathrm{NP}}(\tau, \mu) \quad \text { (at QCD level) }
\end{aligned}
$$

As the final step, any ignorance about the contributions of higher states to the spectral densities may be swept under the carpet by invoking the assumption of (global) quark-hadron duality [6-8], postulating that above - $\tau$-dependent [9-13] — effective thresholds $s_{\text {eff }}(\tau)$ the solely perturbative contributions to the QCD-level correlator cancel the ones of all excited and continuum states to the hadronic correlator:

$$
\int_{s_{\text {phys }}}^{\infty} \mathrm{d} s \exp (-s \tau) \rho_{\text {cont }}(s) \cong \int_{s_{\text {eff }}(\tau)}^{\infty} \mathrm{d} s \exp (-s \tau) \rho_{\text {pert }}(s, \mu) .
$$

Allowing these cancellations to do their job provides the QCD sum rule for the ground-state hadron $H$ :

$$
f_{H}^{2} \exp \left(-M_{H}^{2} \tau\right)=\int_{s_{\mathrm{th}}}^{s_{\mathrm{eff}}(\tau)} \mathrm{d} s \exp (-s \tau) \rho_{\mathrm{pert}}(s, \mu)+\Pi_{\mathrm{NP}}(\tau, \mu)
$$

Eventually, after determination of the $\tau$ dependence of the effective threshold $s_{\mathrm{eff}}(\tau)$ along the lines of a well-developed [14-18] algorithm, the decay constant $f_{H}$ may be extracted from this QCD sum rule by knowledge of the hadron's mass $M_{H}$, or for the choice $\tau=0$, that is, in the so-called local-duality limit.

\section{Strong hadron decays: QCD sum rules from three-point vertex functions}

Mutatis mutandis, hadron form factors [19,20] may be inferred from a three-point correlation function

$$
\Gamma\left(p, p^{\prime}, q\right) \equiv \int \mathrm{d}^{4} x \mathrm{~d}^{4} y \exp \left(\mathrm{i} p x-\mathrm{i} p^{\prime} y\right)\left\langle\Omega\left|\mathrm{T}\left(j(x) J(0) j^{\prime}(y)\right)\right| \Omega\right\rangle
$$

of three currents, $j(x), j^{\prime}(x)$, and $J(x)$, interpolating three hadrons, $H, H^{\prime}$, and $H_{J}$, with decay constants

$$
\langle\Omega|j(0)| H\rangle \propto f_{H}, \quad\left\langle\Omega\left|j^{\prime}(0)\right| H^{\prime}\right\rangle \propto f_{H^{\prime}}, \quad\left\langle\Omega|J(0)| H_{J}\right\rangle \propto f_{H_{J}},
$$




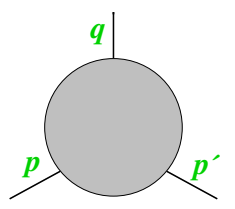

Figure 1. Three-point vertex function $\Gamma\left(p, p^{\prime}, q\right)$ of three hadrons with momenta $p, p^{\prime}$, and $q$.

masses $M_{H}, M_{H^{\prime}}, M_{H_{J}}$, and momenta $p, p^{\prime}, q$, respectively. Their corresponding (simple) kinematics is illustrated by Fig. 1. For all external momenta timelike, i.e., for $p^{2}>0, p^{\prime 2}>0$, and $q^{2}>0$, this vertex function develops a triple pole. One of these poles we attribute to a form factor $F\left(q^{2}\right)$ of the hadron $H_{J}$. The residue of the latter pole at $q^{2}=M_{H_{J}}^{2}$ is defined by the strong coupling $g_{H H^{\prime} H_{J}}$ of the three hadrons:

$$
\Gamma\left(p, p^{\prime}, q\right)=\frac{f_{H}}{p^{2}-M_{H}^{2}} \frac{f_{H^{\prime}}}{p^{\prime 2}-M_{H^{\prime}}^{2}} F\left(q^{2}\right)+\cdots, \quad F\left(q^{2}\right)=\frac{f_{H_{J}}}{q^{2}-M_{H_{J}}^{2}} g_{H H^{\prime} H_{J}}+\cdots .
$$

Again, complex analysis puts us in a position to construct a representation of this three-point correlator $\Gamma\left(p, p^{\prime}, q\right)$ in form of a double dispersion integral of the appropriate double spectral density $\Delta\left(s, s^{\prime}, q^{2}\right)$ :

$$
\Gamma\left(p, p^{\prime}, q\right)=\int \frac{\mathrm{d} s}{s-p^{2}} \frac{\mathrm{d} s^{\prime}}{s^{\prime}-p^{\prime 2}} \Delta\left(s, s^{\prime}, q^{2}\right) .
$$

A double Borel transformation (from the momentum variables $p^{2}$ and $p^{\prime 2}$ to the Borel parameters $\tau$ and $\tau^{\prime}$, respectively) [19-21] and the assumption that quark-hadron duality holds in both the $H$ system and the $H^{\prime}$ system above continuum thresholds $s_{\text {eff }}$ and $s_{\text {eff }}^{\prime}$, respectively, yield the Borelized QCD sum rule

$$
\exp \left(-M_{H}^{2} \tau\right) \exp \left(-M_{H^{\prime}}^{2} \tau^{\prime}\right) F\left(q^{2}\right)=\int^{s_{\text {eff }}} \mathrm{d} s \exp (-s \tau) \int^{s_{\text {eff }}^{\prime}} \mathrm{d} s^{\prime} \exp \left(-s^{\prime} \tau^{\prime}\right) \Delta\left(s, s^{\prime}, q^{2}\right)
$$

By focusing to the series expansion of the perturbative part $\Gamma_{\text {pert }}\left(p^{2}, p^{\prime 2}, q^{2}\right)$ of the correlator $\Gamma\left(p, p^{\prime}, q\right)$

$$
\Gamma_{\text {pert }}\left(p^{2}, p^{\prime 2}, q^{2}\right)=\Gamma_{0}\left(p^{2}, p^{\prime 2}, q^{2}\right)+\alpha_{\mathrm{s}} \Gamma_{1}\left(p^{2}, p^{\prime 2}, q^{2}\right)+O\left(\alpha_{\mathrm{s}}^{2}\right)
$$

in powers of the strong coupling $\alpha_{\mathrm{s}}$, let us now use this example to try to work out the differences in the QCD sum-rule analysis of ordinary hadrons, on the one hand, and of exotic hadrons, on the other hand, as well as to cast some light on difficulties encountered in such studies of strong decays of exotic states.

\section{Correlators of ordinary hadrons: strong three-ordinary-meson couplings}

Ordinary mesons, regarded as bound states of a quark and an antiquark, can be interpolated by suitable quark-antiquark currents $j(x)$ defined in terms of quark fields $q_{i}(x)$ and generalized Dirac matrices $\Gamma_{A}$ :

$$
j(x) \equiv: \bar{q}_{i}(x) \Gamma_{A} q_{j}(x):, \quad q_{i, j}=u, d, s, \ldots, \quad A=1,2, \ldots, 16 .
$$
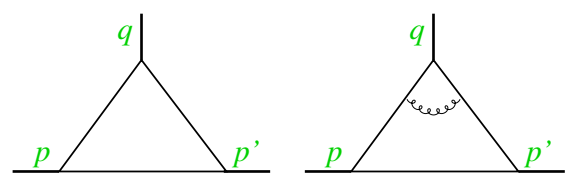

Figure 2. Perturbative expansion of three-point correlation functions $\Gamma\left(p, p^{\prime}, q\right)$ of bilinear quark currents interpolating ordinary mesons: leading-order Feynman diagram (left) vs. one of the next-to-leading-order Feynman diagrams (right).

Inspection of the perturbative expansion (Fig. 2) shows that the leading-order spectral density and thus the form factor $F\left(q^{2}\right)$ receive nonzero contributions from the one-loop lowest-order Feynman diagram, which already provide the bulk of the total three-point correlator [22-27], whereas the contributions of higher-order Feynman diagrams [28-31] stay non-negligible, especially in the large- $q^{2}$ range [32-34]. 


\section{Correlators of one exotic and two ordinary hadrons: exotic-meson decay}

For states composed of four quarks, more precisely, of two quarks and two antiquarks, the construction of operators that interpolate such states is by no means unambiguous. A given four-quark exotic meson can be interpolated by, for instance, tetraquark interpolating operators $\mathcal{T}(x)$ constructed from products of two quark-antiquark currents $\mathcal{J}_{i j}(x)$, which, in turn, are defined by use of appropriate combinations, generically labelled $\mathfrak{M}$, of Dirac and colour matrices and covariant derivatives of the quark fields $q_{i}(x)$ :

$$
\mathcal{T}(x) \equiv: \mathcal{J}_{12}(x) \mathcal{J}_{34}(x):, \quad \mathcal{J}_{i j}(x) \equiv \bar{q}_{i}(x) \mathfrak{M} q_{j}(x), \quad q_{i}=u, d, s, \ldots
$$

The strong decays of any four-quark state, created from the QCD vacuum $|\Omega\rangle$ by this operator, into two ordinary mesons can be analysed by investigation of the three-point correlation function $\Gamma^{j j \mathcal{T}}(x, y, z)$ of two currents $j(x)$ interpolating the generated ordinary mesons and one of our tetraquark currents $\mathcal{T}(x)$ :

$$
\Gamma^{j j \mathcal{T}}(x, y, z) \equiv\langle\Omega|\mathrm{T}(j(x) j(y) \mathcal{T}(z))| \Omega\rangle .
$$

The QCD-level evaluation of this correlation function reveals a crucial difference in the QCD sum-rule description of the characteristics of ordinary hadrons, on the one hand, and exotic hadrons, on the other hand: By arguments based on diagrammatic analysis, it is straightforward to convince oneself that, in a perturbative expansion of the corresponding momentum-space three-point correlator $\Gamma_{\mathrm{QCD}}^{j j \mathcal{T}}\left(p^{2}, p^{\prime 2}, q^{2}\right)$ in powers of the strong coupling $\alpha_{\mathrm{S}}$ (cf. Fig. 3), the lowest-order contribution $\Gamma_{0}^{j j \mathcal{T}}\left(p^{2}, p^{\prime 2}, q^{2}\right)$, of order $O\left(\alpha_{\mathrm{s}}^{0}\right)$, factorizes into the two-point correlation functions $\Pi\left(p^{2}\right)$, i.e., exhibits the factorization property

$$
\Gamma_{0}^{j j \mathcal{T}}\left(p^{2}, p^{\prime 2}, q^{2}\right)=\Pi\left(p^{\prime 2}\right) \Pi\left(q^{2}\right) .
$$

Consequently, the lowest-order contribution arises from disconnected diagrams. ${ }^{1}$ Connected diagrams start to contribute to the correlator $\Gamma_{\mathrm{QCD}}^{j j \mathcal{T}}\left(p^{2}, p^{\prime 2}, q^{2}\right)$ at perturbative order $O\left(\alpha_{\mathrm{s}}\right)$ or higher; this suggests to isolate the disconnected term from all connected contributions $\Gamma_{\text {conn }}^{j j \mathcal{T}}\left(p^{2}, p^{\prime 2}, q^{2}\right)$ to $\Gamma_{\mathrm{QCD}}^{j j \mathcal{T}}\left(p^{2}, p^{\prime 2}, q^{2}\right)$ :

$$
\Gamma_{\mathrm{QCD}}^{j j \mathcal{T}}\left(p^{2}, p^{\prime 2}, q^{2}\right)=\Pi\left(p^{\prime 2}\right) \Pi\left(q^{2}\right)+\alpha_{\mathrm{s}} \Gamma_{\mathrm{conn}}^{j j \mathcal{T}}\left(p^{2}, p^{\prime 2}, q^{2}\right)
$$
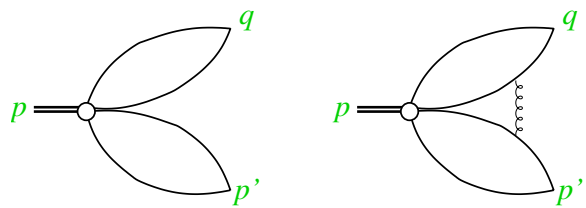

Figure 3. Perturbative expansion of three-point correlation functions $\Gamma_{\mathrm{QCD}}^{j j \mathcal{T}}\left(p^{2}, p^{\prime 2}, q^{2}\right)$ of one exotic tetraquark current $\mathcal{T}$ and two ordinary bilinear quark currents $j$ : lowest-order (left) vs. one of the next-to-leading-order (right) diagrams.

Unfortunately, however, disconnected diagrams bear no relationship at all to the bound states in the focus of our interest; thus, they cannot serve the purpose of acquiring reliable information. ${ }^{2}$ Therefore, studies aiming at the extraction of any features of exotic mesons, such as decay amplitudes, from QCD sum rules that rely exclusively on the factorizable leading-order contribution to the relevant correlation function must be doomed to fail. For some of all the attempts in this latter direction, see Refs. [39-43]. Consequently, the investigation of exotic states by QCD sum rules necessitates the consideration of the contributions of connected diagrams, arising, for exotic states, at higher-than-trivial perturbative order.

\footnotetext{
${ }^{1}$ This result is corroborated by realizing that, upon Borel transformation with respect to the exotic-hadron momentum $p^{2}$, the leading-order, disconnected contribution $\Gamma_{0}^{j j \mathcal{T}}\left(p^{2}, p^{\prime 2}, q^{2}\right)$ to the three-point correlator $\Gamma_{\mathrm{QCD}}^{j j \mathcal{T}}\left(p^{2}, p^{\prime 2}, q^{2}\right)$ gets mapped onto zero.

${ }^{2}$ For related considerations targeting the limit of the number of colours, $N_{\mathrm{c}}$, becoming large, $N_{\mathrm{c}} \rightarrow \infty$, consult Refs. [35-38].
} 


\section{Musings: prospects of elucidation of internal structure of exotic mesons}

Depending on the Lorentz nature of the employed operators encoded in generalized Dirac matrices $\Gamma_{A}$, $A=1,2, \ldots, 16$, an ordinary meson $M$ may be interpolated by only a comparatively small set of (linear combinations of) bilinear quark currents $j_{A}(x)$ and therefore has only one or a few decay constants $f_{M_{A}}$ :

$$
\left\langle\Omega\left|j_{A}(0)\right| M\right\rangle \propto f_{M_{A}}, \quad j_{A}(x) \equiv: \bar{q}_{1}(x) \Gamma_{A} q_{2}(x):, \quad q_{i, j}=u, d, s, \ldots
$$

In contrast to this, an exotic hadron may be interpolated by a whole plethora of interpolating operators: for instance, a tetraquark meson $\Theta$ may be described by products of two quark-antiquark currents $J(x)$,

$$
\langle\Omega|\mathcal{M}(0)| \Theta\rangle \propto f_{\mathcal{M}}, \quad \mathcal{M}(x) \equiv: J_{12}(x) J_{34}(x):, \quad J_{i j}(x) \equiv \bar{q}_{i}(x) \Gamma_{A} q_{j}(x),
$$

or of a diquark current $\mathcal{A}^{a}(x)$ and an antidiquark current $\overline{\mathcal{A}}^{a}(x)$, carrying colour indices $a, b, c=1,2,3$,

$$
\langle\Omega|\mathcal{D}(0)| \Theta\rangle \propto f_{\mathcal{D}}, \quad \mathcal{D}(x) \equiv: \mathcal{A}_{12}^{a}(x) \overline{\mathcal{A}}_{34}^{a}(x):, \quad \mathcal{A}_{i j}^{a}(x)=\epsilon_{a b c} q_{i, b}^{\mathrm{T}}(x) \Gamma_{A} q_{j, c}(x) .
$$

Accordingly, a tetraquark meson $\Theta$ may be characterized by a large number of decay constants defined by the four-quark interpolating currents $\mathcal{T}(x), \mathcal{M}(x), \mathcal{D}(x)$ and providing clues to its internal structure:

- On the one hand, if all members $f_{\mathcal{M}}$ of the set of decay constants caused by quark-antiquark currents prove to be of low numerical relevance, $f_{\mathcal{M}} \approx 0 \forall \mathcal{M}$, we may be tempted to call the exotic meson $\Theta$ a colour-neutral, of course, bound state of a colour-triplet diquark and a colour-antitriplet antidiquark.

- On the other hand, if all elements $f_{\mathcal{D}}$ of the set of decay constants of (anti-)diquark-current origin are comparatively tiny, $f_{\mathcal{D}} \approx 0 \forall \mathcal{D}$, we will tend to regard the exotic meson $\Theta$ as a molecular-type bound state built up by two colourless mesons created from the vacuum by their interpolating currents $J(x)$.

Needless to say, any operator with the appropriate or desired quantum numbers may be adopted for the interpolation of, and can therefore potentially contribute to, the hadronic state under consideration. An exotic meson, for example, might be found to consist of both a two-quark and a four-quark component. In addition, Fierz transformations offer the possibility to reshuffle the order of the quark field operators in linear combinations of four-quark interpolating currents and to switch between diquark-antidiquark and meson-meson structures [44]. All this contributes to blurring the emerging picture of exotic states.

Theoretically, decay constants of the kind introduced above may be approached by QCD sum rules based on the two-point correlation functions of suitable tetraquark interpolating operators $\theta(x)[45,46]$

$$
\left\langle\Omega\left|\mathrm{T}\left(\theta(x) \theta^{\dagger}(0)\right)\right| \Omega\right\rangle, \quad \theta(x) \in\{\mathcal{T}(x), \mathcal{M}(x), \mathcal{D}(x), \ldots\} .
$$

In this context, we face, however, the problem that, similar to the case of three-point correlators, not all contributions to these two-point correlation functions are related to the properties of the corresponding exotic states. Notwithstanding this, we are confident [47] that the QCD sum-rule formalism enables us to derive exotic-hadron decay constants in a similar manner as in the case of ordinary hadrons [48-52].

\section{Acknowledgements}

D. M. would like to express gratitude for support by the Austrian Science Fund (FWF) under project P29028-N27. 


\section{References}

[1] M. A. Shifman, A. I. Vainshtein, and V. I. Zakharov, Nucl. Phys. B 147 (1979) 385.

[2] P. Colangelo and A. Khodjamirian, in At The Frontier of Particle Physics - Handbook of QCD, edited by M. Shifman (World Scientific, Singapore, 2001), Vol. 3, p. 1495, arXiv:hep-ph/ 0010175.

[3] B. L. Ioffe, Prog. Part. Nucl. Phys. 56 (2006) 232, arXiv:hep-ph/0502148.

[4] K. G. Wilson, Phys. Rev. 179 (1969) 1499.

[5] V. A. Novikov, M. A. Shifman, A. I. Vainshtein, and V. I. Zakharov, Nucl. Phys. B 249 (1985) 445.

[6] B. Blok, M. Shifman, and D.-X. Zhang, Phys. Rev. D 57 (1998) 2691, arXiv:hep-ph/9709333; 59 (1999) 019901(E).

[7] M. Shifman, in At The Frontier of Particle Physics - Handbook of QCD, edited by M. Shifman (World Scientific, Singapore, 2001), Vol. 3, p. 1447, arXiv:hep-ph/0009131.

[8] W. Lucha and D. Melikhov, Phys. Rev. D 73 (2006) 054009, arXiv:hep-ph/0602217.

[9] W. Lucha, D. Melikhov, and S. Simula, Phys. Rev. D 79 (2009) 096011, arXiv:0902.4202 [hep$\mathrm{ph}$.

[10] W. Lucha, D. Melikhov, and S. Simula, J. Phys. G 37 (2010) 035003, arXiv:0905.0963 [hep-ph].

[11] W. Lucha, D. Melikhov, H. Sazdjian, and S. Simula, Phys. Rev. D 80 (2009) 114028, arXiv: 0910.3164 [hep-ph].

[12] W. Lucha, D. Melikhov, and S. Simula, Phys. Lett. B 687 (2010) 48, arXiv:0912.5017 [hep-ph].

[13] W. Lucha, D. Melikhov, and S. Simula, Phys. At. Nucl. 73 (2010) 1770, arXiv:1003.1463 [hep$\mathrm{ph}]$.

[14] W. Lucha, D. Melikhov, and S. Simula, Phys. Rev. D 76 (2007) 036002, arXiv:0705.0470 [hep$\mathrm{ph}]$.

[15] W. Lucha, D. Melikhov, and S. Simula, Phys. Lett. B 657 (2007) 148, arXiv:0709.1584 [hep-ph].

[16] W. Lucha, D. Melikhov, and S. Simula, Phys. At. Nucl. 71 (2008) 1461.

[17] W. Lucha, D. Melikhov, and S. Simula, Phys. Lett. B 671 (2009) 445, arXiv:0810.1920 [hep-ph].

[18] D. Melikhov, Phys. Lett. B 671 (2009) 450, arXiv:0810.4497 [hep-ph].

[19] B. L. Ioffe and A. V. Smilga, Phys. Lett. B 114 (1982) 353.

[20] V. A. Nesterenko and A. V. Radyushkin, Phys. Lett. B 115 (1982) 410.

[21] A. P. Bakulev and A. V. Radyushkin, Phys. Lett. B 271 (1991) 223.

[22] I. Balakireva, W. Lucha, and D. Melikhov, J. Phys. G 39 (2012) 055007, arXiv:1103.3781 [hep$\mathrm{ph}]$.

[23] W. Lucha and D. Melikhov, J. Phys. G 39 (2012) 045003, arXiv:1110.2080 [hep-ph].

[24] I. Balakireva, W. Lucha, and D. Melikhov, Phys. Rev. D 85 (2012) 036006, arXiv:1110.6904 [hep-ph].

[25] D. Melikhov and B. Stech, Phys. Rev. D 85 (2012) 051901(R), arXiv:1202.4471 [hep-ph].

[26] W. Lucha and D. Melikhov, Phys. Rev. D 86 (2012) 016001, arXiv:1205.4587 [hep-ph].

[27] D. Melikhov and B. Stech, Phys. Lett. B 718 (2012) 488, arXiv:1206.5764 [hep-ph].

[28] E. Bagan, P. Ball, and P. Gosdzinsky, Phys. Lett. B 301 (1993) 249, arXiv:hep-ph/9209277.

[29] P. Colangelo, F. De Fazio, and N. Paver, Phys. Rev. D 58 (1998) 116005, arXiv:hep-ph/9804377.

[30] V. V. Braguta and A. I. Onishchenko, Phys. Lett. B 591 (2004) 255, arXiv:hep-ph/0311146.

[31] V. V. Braguta and A. I. Onishchenko, Phys. Lett. B 591 (2004) 267, arXiv:hep-ph/0403240. 
[32] V. Anisovich, D. Melikhov, and V. Nikonov, Phys. Rev. D 52 (1995) 5295, arXiv:hep-ph/ 9503473.

[33] V. V. Anisovich, D. I. Melikhov, and V. A. Nikonov, Phys. Rev. D 55 (1997) 2918, arXiv:hep-ph/ 9607215.

[34] V. Braguta, W. Lucha, and D. Melikhov, Phys. Lett. B 661 (2008) 354, arXiv:0710.5461 [hep$\mathrm{ph}]$.

[35] S. Weinberg, Phys. Rev. Lett. 110 (2013) 261601, arXiv:1303.0342 [hep-ph].

[36] M. Knecht and S. Peris, Phys. Rev. D 88 (2013) 036016, arXiv:1307.1273 [hep-ph].

[37] T. D. Cohen and R. F. Lebed, Phys. Rev. D 89 (2014) 054018, arXiv:1401.1815 [hep-ph].

[38] T. D. Cohen and R. F. Lebed, Phys. Rev. D 90 (2014) 016001, arXiv:1403.8090 [hep-ph].

[39] M. Nielsen, F. S. Navarra, and S. H. Lee, Phys. Rep. 497 (2010) 41, arXiv:0911.1958 [hep-ph].

[40] M. Nielsen and F. S. Navarra, Mod. Phys. Lett. A 29 (2014) 1430005, arXiv:1401.2913 [hep-ph].

[41] Z.-G. Wang and T. Huang, Nucl. Phys. A 930 (2014) 63, arXiv:1312.2652 [hep-ph].

[42] J. M. Dias et al., Phys. Lett. B 758 (2016) 235, arXiv:1603.02249 [hep-ph].

[43] M. Nielsen et al., arXiv:1611.03300 [hep-ph], these proceedings.

[44] R. L. Jaffe, Nucl. Phys. A 804 (2008) 25.

[45] F. Fanomezana, S. Narison, and A. Rabemananjara, Nucl. Part. Phys. Proc. 258-259 (2015) 152, arXiv:1409.8591 [hep-ph].

[46] R. Albuquerque, S. Narison, A. Rabemananjara and D. Rabetiarivony, Int. J. Mod. Phys. A 31 (2016) 1650093, arXiv:1604.05566 [hep-ph].

[47] W. Lucha and D. Melikhov, arXiv:1608.06873 [hep-ph].

[48] W. Lucha, D. Melikhov, and S. Simula, J. Phys. G 38 (2011) 105002, arXiv:1008.2698 [hep-ph].

[49] W. Lucha, D. Melikhov, and S. Simula, Phys. Lett. B 701 (2011) 82, arXiv:1101.5986 [hep-ph].

[50] W. Lucha, D. Melikhov, and S. Simula, Phys. Rev. D 88 (2013) 056011, arXiv:1305.7099 [hep$\mathrm{ph}]$.

[51] W. Lucha, D. Melikhov, and S. Simula, Phys. Lett. B 735 (2014) 12, arXiv:1404.0293 [hep-ph].

[52] W. Lucha, D. Melikhov, and S. Simula, Phys. Rev. D 91 (2015) 116009, arXiv:1504.03017 [hep-ph]. 